21. Saunders, W., Rowan-Robinson, M., Lawrence, A., et al. The 60-micron and far-infrared luminosity functions of IRAS galaxies // Mon. Not. Roy. Astron. Soc. - 1990. - V.242. -P. 318-337.

22. Schechter, P. An analytic expression for the luminosity function for galaxies // Astrophys. J. - 1976. - V. 203. -P. $297-306$.

23. Takeuchi, T. T., Yoshikawa, K., Ishii, T. T. The Luminosity Function of IRAS Point Source Catalog Redshift Survey Galaxies // Astrophys. J. - 2003. - V. 587, Issue 2. - P. L89-L92.

24. Willott, C. J., Rawlings, S., Blundell, K. L., et al. The radio luminosity function from the low-frequency 3 CRR, 6 CE and $7 C R S$ complete samples // Mon. Not. Roy. Astron. Soc. 2001. -V. 322, Issue 3. - P. 536-552.

25. Wyder, T. K., Treyer, M. A., Milliard, B., et al. The Ultraviolet Galaxy Luminosity Function in the Local Universe from GALEX Data // Astrophys. J. - 2005. - V. 619, Issue 1. - P. L15-L18.

Надійшла до редколегії 27.04.16

S. Parnovsky, Dr. Sci,

I. Izotova, Ph. D

Astronomical Observatory of National Taras Shevchenko University of Kyiv

\title{
INITIAL LUMINOSITY FUNCTIONS OF STARBURST GALAXIES
}

For the sample of about 800 starburst galaxies the initial luminosity functions which appear the distributions of galaxy luminosities at zero starburst age are considered based on the data of luminosities of galaxies in the recombination Ha emission line in the regions of ionised hydrogen and the ultraviolet continuum. We find the initial luminosity functions for the starburst galaxies with Ha emission and ultraviolet continuum are satisfactory approximated with log-normal function.

С. Парновский, д-р физ.-мат. наук,

И. Изотова, канд. физ.-мат. наук

Астрономическая обсерватория Киевского национального университета

имени Тараса Шевченко

\section{НАЧАЛЬНЫЕ ФУНКЦИИ СВЕТИМОСТИ ГАЛАКТИК С АКТИВНЫМ ЗВЕЗДООБРАЗОВАНИЕМ}

На основе данных об излучении выборки 800 галактик с активным звездообразованием в рекомбинационной линии На в зонах ионизованного водорода и ультрафиолетовом континууме рассмотрены начальные функции светимости, которые описывают распределение светимостей галактик при нулевом возрасте вспышки звездообразования. Показано, что начальные функции светимости галактик с активным звездообразованием в рассмотренных диапазонах могут быть удовлетворительно представлены lоgнормальной функцией, которая удовлетворительно описывает и текущие функции светимости.

УДК 524.7

В. Жданов, д-р. фіз.-мат. наук, проф., С. Дилда, студ. фіз. ф-ту,

Київський національний університет імені Тараса Шевченка

\section{ЯКІСНИЙ АНАЛІЗ ЕВОЛЮЦІЇ ВСЕСВІТУ В ГІДРОДИНАМІЧНІЙ МОДЕЛІ 3 БАРОТРОПНИМ РІВНЯННЯМ СТАНУ}

Досліджено якісну поведінку масштабного фактора та густини енергії в гідродинамічній моделі однорідного ізотропного Всесвіту з загальним баротропним рівнянням стану (PC). Аналіз проведено за умов на РС більш загальних, ніж це було зроблено раніше у стаmmi [Jenkovszky et al., Phys. Rev. D 90, 023529 (2014)], зокрема, допускаються випадки з двома чи більше точками, де питома ентальпія космологічної рідини дорівнює нулю. Подано якісну класифікацію, яка включає можливі сценарії космологічної еволюції з асимптотично експоненційною інфляцією, аналоги "Великого розриву" у майбутньому чи у минулому, розв'язки без сингулярностей та осцилюючі всесвіти.

1. Вступ. Сучасна космологічна $\Lambda$ CDM-модель описує практично усі спостережні дані позагалактичної астрономії. Тим не менш, відомі проблеми горизонту та плоскостності вимагають шукати інші підходи для опису перших миттєвостей після Великого вибуху, пов'язаних з інфляційною стадією (див., напр., [1-4]). У сучасній космології відома низка явних аналітичних розв'язків, що описують еволюцію однорідного ізотропного Всесвіту. Але досить часто на перший план виходять якісні властивості космологічних сценаріїв, а саме: чи існує розв'язок для усіх часів, починаючи від космологічної сингулярності або він означений на скінченному інтервалі, коли прямують до нескінченності густина енергії або тиск і за яких умов тощо (див., напр., огляди в [5-8]). 3 цією метою в [8] було досліджено якісну поведінку розв'язків в гідродинамічній моделі однорідного ізотропного Всесвіту; баротропне рівняння стану, що розглядалося, було досить загальним, тим не менш, були застосовані певні обмеження щодо монотонності деяких складових цього рівняння. У даній роботі ми знімаємо ці обмеження і розглядаємо баротропне рівняння стану $p=p(e)$, яке пов'язує тиск $p$ та інваріантну густину енергії $е$ космологічної рідини лише за загальних умов однозначності та гладкості. Далі буде описано можливі сценарії космологічної еволюції в рамках цієї моделі в залежності від наявності коренів питомої ентальпії $h(e)=e+p(e)$, яку вважаємо гладкою функцією за усіх значень аргументу. Також припускатимемо, що $h(0)=0$.

2. Основні рівняння. Метрику простору-часу в однорідній ізотропній космології можна записати так:

$$
d s^{2}=d t^{2}-a^{2}(t)\left[d \chi^{2}+F^{2}(\chi) d O^{2}\right]
$$

де $F(x)=\sin (x), \sinh (x)$ або $x$ в залежності від параметру $k$, що може мати значення відповідно $1,-1$ або 0 (замкнений, відкритий або просторово-плоский Всесвіт).

Рівняння Фрідмана, що мають вид:

$$
\frac{d^{2} a}{d t^{2}}=-\frac{4 \pi}{3} a(e+3 p)
$$




$$
H^{2}=\frac{8 \pi}{3} e-\frac{k}{a^{2}}, \quad H=a^{-1} d a / d t,
$$

розглядають разом із рівнянням релятивістської гідродинаміки

$$
\frac{d e}{d t}+3 h H=0 ;
$$

втім, ці рівняння не є незалежними

Рівняння (4) можна переписати у вигляді автономного рівняння першого порядку

$$
\frac{d e}{d X}=-3 h, \quad X=\ln a .
$$

Якщо маємо точку $e_{1}: \quad h\left(e_{1}\right)=0$, то $e(X) \equiv e_{1} €$ розв'язком рівняння (5) і в силу теореми єдиності жоден інший розв'язок $e(X)$ цього рівняння не може перетнути значення $e_{1}$. Це дає змогу дати простий розгляд якісної поведінки можливих розв'язків.

3. Аналіз розв'язків рівняння (5). Проаналізуємо поведінку розв'язків рівнянь (1-4) в залежності від розташування коренів функції $h(e)$. Спочатку розглянемо допустимі неперервно-диференційовні розв'язки (4) або (5). Нехай $e_{1} \geq 0$ - це максимальний з усіх коренів функції $h(e)$, причому $h(e)>0$ при $e>e_{1}$. Тоді в області $e>e_{1}$ на площині $(X, e)$ можливі лише такі типи поведінки розв'язків рівняння (5).

U1 $\downarrow$. $e(X)$ визначена і монотонно спадає від нескінченності до $e_{1}$ на усій дійсній $X$-осі $e(X) \rightarrow \infty, X \rightarrow-\infty$; $e(X) \rightarrow e_{1}, X \rightarrow \infty$. Простий приклад такої поведінки маємо у випадку $p(e)=w e, w=c o n s t>-1$. Варіант, коли $e(X)<C<\infty \forall X$ неможливий, оскільки у цьому випадку має існувати $e_{*}$, таке, що $e(X) \rightarrow e_{*}>e_{1}$ за $X \rightarrow-\infty$, звідки $h\left(e_{*}\right)=0$, що суперечить умові $h\left(e_{*}\right)>0$. Аналогічно виключаємо варіант, коли $\exists e_{2}: e(X) \rightarrow e_{2}>e_{2}, X \rightarrow \infty$. Варіант, коли функція $e(X) €$ обмеженою на обмеженому інтервалі, але не подовжується за його межі, суперечить теоремам існування та єдиності (подібні якісні міркування, основані на базових властивостях діференціальних рівнянь першого порядку для однієї функції, систематично використовуються надалі без деталізації). Натомість, можливий випадок. коли $e(X) \in$ необмеженою на обмеженому інтервалі:

U2 $\downarrow$ : $e(X)$ визначена і монотонно спадаюча за $X>X_{0}>-\infty, e(X) \rightarrow \infty, X \rightarrow X_{0}+0, e(X) \rightarrow e_{1}, X \rightarrow \infty$. Розв'язок (5) не може бути подовжений неперервним чином на значення, менші за $X_{0}$.

Приклад відповідного рівняння стану: $p(e)=w e+\alpha e^{1+\varepsilon}, w>-1, \alpha>0, \varepsilon>0-$ константи.

Нехай тепер $h(e)<0$ при $e>e_{1}$, де $e_{1} \geq 0$ - це максимальний з усіх коренів функції $h(e)$. Аналогічно попередньому маємо, що в області $е>e_{1}$ можливі лише такі типи поведінки розв'язків рівняння (5).

U1个. $e(X)$ визначена і монотонно зростаюча від $e_{1}$ до нескінченності на усій дійсній $X-\infty$ осі, $e(X) \rightarrow \infty, X \rightarrow \infty$; $e(X) \rightarrow e_{1}, X \rightarrow-\infty$.

U2 $\uparrow$. $e(X)$ визначена і монотонно зростаюча, але область зміни аргументу обмежена: $X<X_{0}<\infty$, $e(X) \rightarrow e_{1}, X \rightarrow-\infty ; e(X) \rightarrow \infty, X \rightarrow X_{0}-0$.

B $\downarrow$. Нехай $h\left(e_{1}\right)=h\left(e_{2}\right)=0,0 \leq e_{1}<e_{2}<\infty$ та $h(e)>0$ на інтервалі $e \in\left(e_{1}, e_{2}\right)$. Тоді на площині $(X, e)$ в області $e \in\left(e_{1}, e_{2}\right)$, розв'язок (5) $e(X)$ визначений, обмежений і монотонно спадає на усій дійсній осі, $e(X) \rightarrow e_{1}, X \rightarrow-\infty$; $e(X) \rightarrow e_{2}, X \rightarrow \infty$.

$\mathbf{B} \uparrow$. Нехай $h\left(e_{1}\right)=h\left(e_{2}\right)=0,0 \leq e_{1}<e_{2}<\infty$ та $h(e)<0$ на інтервалі $e \in\left(e_{1}, e_{2}\right)$. Тоді в області $e \in\left(e_{1}, e_{2}\right)$ розв'язок (5) $e(X)$ визначений, обмежений й монотонно зростає на усій дійсній осі, $e(X) \rightarrow e_{2}, X \rightarrow-\infty ; e(X) \rightarrow e_{1}, X \rightarrow \infty$.

4. Космологічні сценарії при $\boldsymbol{k}=\mathbf{0},-1.3$ рівняння (3)

$$
\frac{d X}{d t}=s \sqrt{\frac{8 \pi}{3} e-k \exp (-2 X)}
$$

де у випадку космологічного розширення $s=1(\dot{a}>0)$ та у випадку стиснення $s=-1(\dot{a}<0)$.

За $k=0,-1$ (відкритий або просторово-плоский Всесвіт), у рівнянні (3) права частина ніколи не обертається на нуль, тобто всюди $а(t)$ або монотонно спадає (стиснення, $s=-1$ ), або монотонно зростає (розширення, $s=1$ ).

4.1. У випадку залежності U1 для $e(X), k=-1, s=1$, з п.3 маємо нескінченне (монотонне) зростання масштабного фактора $a(t) \rightarrow \infty$ та монотонне спадання густини $e(t) \rightarrow e_{1} \geq 0$ при $t \rightarrow \infty$. Зауважимо, що коли $e_{1}>0$, це значення можна ототожнити із сучасним значенням густини темної енергії, яка нині складає приблизно 70\% усієї космологічної густини. Еволюція починається зі скінченного значення часу $t=t_{0}>-\infty$. Дійсно, якщо від протилежного припустити, що $a(t)>0, e(t)>0$ існують при усіх $t \rightarrow-\infty$, з рівняння (6) маємо $\frac{d X}{d t}>\exp (-X)$. 3 цієї диференціальної нерівності легко 
отримати, що $X(t)$ при зменшенні $t$ обов'язково натрапляє на сингулярність за деякого скінченного $t=t_{1}>-\infty$, а це суперечить припущенню.

Значення $t_{0}$ можуть бути різними для різних розв'язків, але вибір початку відліку часу дозволяє покласти $t_{0}=0$; далі так і будемо робити. Таким чином, у випадку U1 $\downarrow$ маємо $a(t): 0 \uparrow \infty, e(t): \infty \downarrow e_{0}$ на $(0, \infty) \quad\left(д а л і ~ f: a_{1} \uparrow a_{2}\right.$ означає, що деяка функція $f$ монотонно зростає від $a_{1}$ до $a_{2}$, прямуючи до цих значень на межах області означення. Аналогічно $a_{1} \downarrow a_{2}$ у випадку спадання. Варіант $f: a_{1} \uparrow a_{2} \downarrow a_{1}$ означає, що зростання від $a_{1}$ до $a_{2}\left(a_{1}<a_{2}\right)$ після досягнення точки повороту $a_{2}$ змінюється на спадання до $\left.a_{1}\right)$. У випадку U2 $\downarrow$ маємо аналогічну поведінку, але $a(t): a_{0} \uparrow \infty$ зростає на $(0, \infty)$ не від нуля, а від деякого значення $a(0)=a_{0}=\exp \left(X_{0}\right)>0$. Це значення також може бути різним для різних розв'язків, але воно обов'язково існує для будь-якого розв'язку у випадку U2 $\downarrow$.

4.2. Нехай $h(e)<0, s=1$. У випадку U1个, $k=-1$, а(t) зростає на $t \in\left(0, t_{*}\right)$ від 0 до $\infty$, де $t_{*} \leq \infty$, тобто значення $t_{*}$ може бути і скінченним ("Big Rip"[8]) і нескінченним; також $e(t): e_{1} \uparrow \infty$. Для U2 $\uparrow$ маємо аналогічну поведінку, але тут існує максимальне $a_{0}=\exp \left(X_{0}\right)<\infty$, таке, що $a(t): 0 \uparrow a_{0}$, причому $e(t) \rightarrow \infty$ при $a(t) \rightarrow a_{0}$.

4.3. У випадках $\mathrm{B} \downarrow, \mathbf{B} \uparrow, s=1$, маємо $t \in(0, \infty), a(t): 0 \uparrow \infty$, густина енергії або зростає (B $\uparrow)$, або спадає (B $\downarrow)$ на $\left(e_{1}, e_{2}\right)\left(0 \leq e_{1}<e_{2}<\infty\right)$.

4.4. При $k=0, s=+1$ можливими $\epsilon$, по-перше, ті ж самі варіанти областей існування та областей зміни фрункцій $a(t), e(t)$, як у $4.1-4.3$ у випадку $k=-1, s=+1$. По-друге, для U1 $\downarrow$ додатково можливі випадки, коли розв'язок існує на

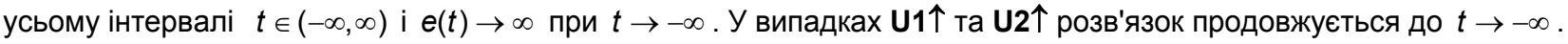

Розв'язки, що відповідають стисненню Всесвіту $(s=-1)$ для $k=0,-1$ отримуємо з попереднього розгляду заміною $t \rightarrow-t$.

5. Космологічні сценарії при $\boldsymbol{k}=1$. При $k=1$ еволюція залежить від наявності нулів фуннції $F(X)=8 \pi e(X) / 3-\exp (-2 X)$. Якщо це нулі прості, вони будуть точками повороту для $X(t)$, тобто при переході через цю точку відбувається зміна між режимами розширення та стиснення (зміни знаку $s$ ). Зауважимо, що коли $F\left(X_{0}\right)=0$, формальний розв'язок $a(t) \equiv \exp \left(X_{0}\right)$ рівняння (6) або рівняння (3) описує космологічну еволюцію тоді й тільки тоді, коли $e+3 p \equiv 0$, або, еквівалентно, $F^{\prime}\left(X_{0}\right)=0$.

Маємо такі варіанти розташування нулів функції $F(X)$ та, відповідно, поведінки функцій $а(t), e(t)$.

5.1. Нехай $s=1$, в області $e>e_{0} \geq 0$, де $h(e)>0, h\left(e_{0}\right)=0$, маємо $F(X)>0$ для усіх $X$ з області означення $e(X)$. Тут можливі усі варіанти поведінки, аналогічні п.4.4, у тому числі, коли інтервал $t$, де визначений розв'язок, починається $3-\infty$, або коли еволюція масштабного фактора починається 3 деякого додатного значення: $a(t)>a_{0}>0$. Протилежний випадок стиснення $(s=-1)$ отримуємо заміною $t \rightarrow-t$.

5.2. Нехай існує точка повороту $X_{0}, F(X)>0, \quad X<X_{0}<\infty ; \quad F\left(X_{0}\right)=0, \quad F^{\prime}\left(X_{0}\right) \neq 0$ і в цій області $h(e(X))>0$. Тут розширення переходить у стиснення $(s=1 \rightarrow s=-1)$, наприклад $a(t): 0 \uparrow a_{0} \downarrow 0$. При $t \rightarrow \pm \infty$ можливі варіанти поведінки (аналогічні п.4.4), (i) коли інтервал $t$, де визначений розв'язок, починається з $-\infty$ і закінчується на $-\infty$, (ii) коли інтервал $t$, де визначений розв'язок, починається з 0 і закінчується у деякому $t_{0}<\infty$, причому можливі як варіанти, коли еволюція масштабного фактора починається з деякого додатного значення: $a(t)>a_{1}>0$ і закінчується на ньому, так і варіанти, коли це значення $a_{1}=0$.

5.3. Нехай тепер $\exists X_{0}, \quad F(X)>0 \quad \forall X>X_{0}>-\infty ; \quad F\left(X_{0}\right)=0, \quad F^{\prime}\left(X_{0}\right) \neq 0$. Можливі випадки з відскоком: коли за $h(e)>0$ маємо $a(t): \infty \downarrow a_{0} \uparrow \infty ; \quad a_{0}=\exp \left(X_{0}\right), e(t): e_{1} \uparrow e\left(X_{0}\right) \downarrow e_{1}, t \in(-\infty, \infty)$ та аналогічну поведінку за $h(e)<0, a$ також коли за $h(e)<0$ маємо $a(t): \infty \downarrow a_{0} \uparrow \infty=\exp \left(X_{0}\right)$, але на скінченному інтервалі $t$.

5.4. Нехай існують скінченні корені $X_{0}, X_{1}\left(-\infty<X_{0}<X_{1}<\infty\right)$ функції $F(X)$, такі, що

$F\left(X_{i}\right)=0, F^{\prime}\left(X_{i}\right) \neq 0, i=0,1 ; \quad F(X)>0 \quad X \in\left(X_{0}, X_{1}\right)$. Така ситуація може бути реалізована у випадках U1 $\downarrow$, U2 $\downarrow$, В $\downarrow$. При цьому, очевидно, маємо періодичний розв'язок $а(t), e(t)$.

Якщо зняти одну з умов $F^{\prime}\left(X_{i}\right) \neq 0$, матимемо розв'язок на $t \in(-\infty, \infty)$ з точкою повороту. Якщо $F^{\prime}\left(X_{i}\right)=0$, точки повороту немає, розв'язок монотонний на $t \in(-\infty, \infty)$.

Таким чином, в рамках гідродинамічної моделі однорідного ізотропного Всесвіту за досить загальних умов на баротропне рівняння стану, подано перелік якісних сценаріїв космологічної еволюції з огляду на характер монотонності масштабного фактора та густини енергії, а також у залежності від того, чи є область визначеності та область значень цих функцій обмеженими або необмеженими. Типи розв'язків описують, зокрема, нескінченне зростання параметрів задачі на скінченому або напів-нескінченному інтервалі часу, осцилюючі всесвіти або еволюцію з відскоком.

Публікація містить результати досліджень, проведених при частковій підтримці Державного фронду фундаментальних досліджень за проектом Ф64/45-2016. 
Список використаних джерел

1. Яцків Я.С., Александров О.М., Вавилова І.Б. [та ін.] Загальна теорія відносності: випробування часом.- Київ: ГАО НАН України, 2005.

2. Яцків Я.С., Александров О.М., Вавилова І.Б. [та ін.] Загальна теорія відносності: горизонти випробувань. - Київ: ВАІТЕ, 2013.

3. Александров А.Н., Вавилова И.Б., Жданов В.И. [и др.] Общая теория относительности: признание временем. - К.: Наукова Думка, 2015.

4. Novosyadlyi B., Pelykh V., Shtanov Yu., Zhuk A. Dark energy and dark matter of the universe: in three volumes / Ed. V. Shulga. - Vol. 1: Dark matter: Observational evidence and theoretical models. K.: Akademperiodyka, -2013.

5. Nojiri S., Odintsov S.D. , Tsujikawa S. Properties of singularities in (phantom) dark energy universe // Phys.Rev.D71:063004 (2005).

6. Nojiri S., Odintsov S.D. Modified gravity with negative and positive powers of the curvature: unification of the inflation and of the cosmic acceleration // Phys.Rev.D68:123512 (2003).

7. Bamba K., Odintsov S.D. Universe acceleration in modified gravities: $F(R)$ and $F(T)$ cases// arXiv:1402.7114 [hep-th] (2014).

8. Jenkovszky L.L., Zhdanov V.I., Stukalo E.J. Cosmological model with variable vacuum pressure. Phys.Rev.D90, 023529 (2014).

9. Caldwell R.R., Kamionkowski M., Weinberg N.N. Phantom Energy: Dark Energy with w<-1 Causes a Cosmic Doomsday. Phys.Rev.Lett., vol. 91, Issue 7, id. 071301 (2003).

Надійшла до редколегії 30.11.14

V. Zhdanov, Dr. Sci., Prof.

S. Dylda, stud. phys. fac.,

National Taras Shevchenko University of Kyiv

\section{QUALITATIVE ANALYSIS OF A COSMOLOGICAL EVOLUTION IN A HYDRODYNAMICAL MODEL WITH A BAROTROPIC EQUATION OF STATE}

We study a qualitative behavior of the scale factor and energy density in a hydrodynamical model of a homogeneous isotropic Universe with a general barotropic equation of state (EOS). We relax conditions on EOS as compared with the paper by Jenkovszky et al., [Phys. Rev. D 90,023529 (2014)] and to include the cases of two or more points of zero specific enthalpy. We present a classification of possible scenarios with an asymptotically exponential inflation, analogs of the Big Rip in the future and in the past, singularity free bouncing solutions and oscillating Universes.

В. Жданов, д-р. физ.-мат. наук, проф.,

С. Дылда, студ. физ. ф-та,

Киевский национальный университет имени Тараса Шевченко

\section{КАЧЕСТВЕННЫЙ АНАЛИЗ КОСМОЛОГИЧЕСКОЙ ЭВОЛЮЦИИ В ГИДРОДИНАМИЧЕСКОЙ МОДЕЛИ С БАРОТРОПНЫМ УРАВНЕНИЕМ СОСТОЯНИЯ}

Мы исследуем качественное поведение маситабного фактора и плотности энергии в гидродинамической модели однородной изотропной Вселенной с общим баротропным уравнением состояния (УС). Анализ проводится при условии на УС более общем, чем это было сделано ранее в работе Jenkovszky et al., [Phys. Rev. D 90, 023529 (2014)], в т. ч., допускаются случаи с двумя и более точками, где удельная энтальпия космологической жидкости равна О. Приводится качественная классификация, вклющающая в себя возможные сценарии космологической эволюции с ассимпотически экспоненциальной инфляцией, аналоги "Большого Разрыва" в будущем или в прошлом, решения без сингулярностей и осциллирующие вселенные.

УДК 523.64

В. Пономаренко, канд. фіз.-мат. наук, А. Сімон, інженер 2 кат.,

К. Чурюмов, д-р фріз.-мат. наук, профр. Астрономічна обсерваторія Київського національного університету імені Тараса Шевченка

\section{ПАРАMЕТРИ ПИЛУ ТА ГАЗУ У КОМІ КОМЕТ C/2014 Q2 (LOVEJOY) I C/2013 US10 (CATALINA)}

Представлено результати спостережень і досліджень довгоперіодичних комет C/2014 Q2 (Lovejoy) i C/2013 US10 (Catalina) на основі оптичних спектрів з середньою роздільною здатністю ( $/ \Delta \lambda \approx 1200)$. Спектри були отримані у лютому та грудні 2015 року за допомогою телескопа A3T-14 (D=0.48 м, F= 7.7 м) i спектрографа ACח-9 на спостережній станиії "Лісники" Астрономічної обсерваторії Київського національного університету імені Тараса Шевченка. На основі отриманого спектрального матеріалу була проведена ідентифікація спектральних емісійних смуг. Знайдено деякі фізичні параметри нейтральної газової та пилової кометних атмосфер. Побудовано розподіл загального і відбитого потоку енергії вздовж щілини спектрографа. Обчислено потоки, кількість молекул та газопродуктивність для основних молекулярних емісій, відносну пилопродуктивність.

Особливості спостережень комет C/2014 Q2 (Lovejoy) і C/2013 US10 (Catalina) та обробки їх спектрів. Комета C/2014 Q2 (Lovejoy) - довгоперіодична комета (ДПК), яка була відкрита 17 серпня 2014 р. астрономом Тері Лавджоєм з Бризбена (Австралія) з використанням 0.2-метрового телескопа "Celestron C8" (Шмідта-Касегрена). Комета стала п'ятою, яку відкрив Лавджой. Комета C/2014 Q2 була виявлена при видимій зоряній величині $T=15^{\mathrm{m}}$ в південному сузір'ї Корми [1]. Спостереження комети Lovejoy були виконані нами за допомогою телескопарефлектора АЗТ-14 ( $D=0.48$ м, $F=7.7$ м), підвісного призмового спектрографа АСП-9 $(\lambda / \Delta \lambda \approx 1200)$ та ПЗ3 Starlight SXV-H35 (4008x2672 пікселі, 9x9 мкм/піксель). На момент спостережень, комета C/2014 Q2 знаходилася на геліоцентричній відстані $r=1.32$ а.о., геоцентричній відстані $\Delta=1.09$ а.о., мала інтегральну зоряну величину $T=5.2^{\mathrm{m}}$, кут елонгації складав S-O-T $=78^{\circ}$, фазовий кут S-T-O $=47^{\circ}$. Отримано 12 спектрів комети в спектральному діапазоні $\lambda \Lambda=3600-7600 \AA \AA$. 